\title{
On the Stress Dependence of Subgrain Size
}

\author{
G. H. EDWARD, † M. A. ETHERIDGE, B. E. HOBBS \\ Department of Earth Sciences, Monash University, Clayton, \\ Victoria, 3168, Australia.
}

(Received December 22, 1981; in final form May 24, 1982)

A simple model of the dynamic balance between deformation induced dislocation generation and climb controlled dislocation annihilation in subgrain walls is outlined. This results in a stress-subgrain size relationship which involves various material properties, including the creep stress exponent and the creep diffusivity.

Assuming a fixed slip distance for mobile dislocations, the theory predicts that the subgrain size $(d)$ depends on the stress $(\sigma)$ as $d^{4} \propto \sigma^{-n}$, where $n$ is the creep exponent, and the proportionality constant is dependent on material properties, temperature, and other environmental variables. This theoretical prediction is satisfactorily compared with published experimental results for a variety of materials.

The implications of the environmental dependence of the stress-subgrain size relation with regard to its use as a palaeopiezometer in naturally deformed minerals are discussed.

\section{INTRODUCTION}

Steady state microstructures are widespread in crystalline materials undergoing high temperature deformation, and there is commonly a simple relationship between the flow stress and various microstructural parameters (e.g. dislocation density, subgrain size, recrystallized grainsize) under these conditions (Bird et al., 1969; Young and Sherby, 1973; Takeuchi and Argon, 1976). In recent years, the potential of such relationships as indicators of ancient earth stresses (palaeopiezometers) in rocks with frozen-in microstructures has been examined from both theoretical and empirical points of view (Twiss, 1977; Mercier et al., 1977; White, 1979a, b; Etheridge and Wilkie,

† Now at Department of Materials Engineering, Monash University, Clayton, Victoria, 3168, Australia. 
1981). However, despite some encouraging results, there has been some lack of correspondence between various experimental studies, (Mercier et al., 1977; Christie et al., 1980; Ord, 1981), and difficulties have been encountered in applying theoretical or empirical relationships to naturally deformed rocks (White, 1979b; Weathers et al., 1980; Christie and Ord, 1980; Etheridge and Wilkie, 1981). In particular, an extensive study of naturally deformed and recrystallized quartz led Etheridge and Wilkie (1981) to conclude that the relationship between dynamically recrystallized grainsize and stress was more complex than previously envisaged, and that it should include environmental parameters such as temperature and the thermodynamic activites of chemical species.

Existing models of the relationship between subgrain or recrystallized grainsize and stress are static, based upon energy minimization arguments (Holt, 1970; Twiss, 1977; Gittus, 1979). Our aim in this paper is to develop a dynamic model of subgrain formation during creep, and thus to arrive at a stress/subgrain size relationship that reflects the details of the creep process and includes all variables that affect this process. In addition, since dynamic recrystallization in minerals commonly takes place by the progressive rotation of subgrains during steady state deformation (Hobbs, 1968; Poirier and Nicolas, 1975), such a model should also provide the basis for stress/recrystallized grainsize relationships, and we are currently pursuing this problem.

\section{EXISTING STRESS/SUBGRAIN SIZE MODELS}

It is widely accepted that subgrain size (and cell size in lower temperature deformation) are primarily determined by the stress. Only in a few exceptional cases has any temperature or strain dependence been noted (Takeuchi and Argon, 1976).

The relationship can in general be written as

$$
\frac{\sigma}{G}=K\left(\frac{b}{d}\right)^{p}
$$

where $d=$ cell or subgrain size

$b=$ Burgers vector

$\sigma=$ stress

$G=$ shear modulus

and $\quad p$ and $K$ are constants.

It is commonly found that $p \simeq 1$, but individual investigations have found values of $p$ ranging from 4 for aluminium (Orlovã et al., 1972) to 0.5 for $\mathrm{Fe}-3 \%$ Si (Barret et al., 1966). 
A more general equation than Eq. (1) has been suggested, where an allowance is made for a "friction stress." This can be written

$$
\frac{\sigma-\sigma_{o}}{G}=K_{1}\left(\frac{b}{d}\right)^{q}
$$

where $K_{1}$ and $q$ are constants and $\sigma_{o}$ is the "friction stress," which could be regarded as an empirical constant, and can in principle be determined from polyslip single crystal data (Thompson, 1977). For pure materials, it is expected that $\sigma_{o}$ will be small (Thompson, 1977), in which case Eq. (2) reduces to Eq. (1), perhaps explaining the success of Eq. (1) with most data.

Cell and subgrain walls form because a uniform array of dislocations usually can reduce the total free energy by clustering or polygonization, and existing models of cell or subgrain formation have used an energy minimization approach.

In an attempt to model the formation of cells, Holt (1970) considered a uniform array of screw dislocations, and, using an analysis similar to spinodal decomposition theory, showed that the dislocations should rearrange to form a modulated structure, thus minimizing their elastic interaction energy. He then identified the cell size with the period of modulations. This resulted in a stress/cell size relationship of the form of Eq. (1), with $p=1$, that is,

$$
\frac{\sigma}{G}=K_{2} \frac{b}{d}
$$

where $d=$ cell size

$b=$ Burgers vector

$\sigma=$ stress

$G=$ shear modulus

and $K_{2}=$ constant.

Gittus (1979), also assumed that an initially uniform array of dislocations formed a cell structure to minimize free energy, but his approach differed in a number of physical assumptions. He assumed that the free energy of the material had two components - the elastic free energy (taking account of modulus changes due to dislocation bowing) and the self energy of the dislocations. He also assumed that all of the dislocations were arranged into cell walls. Gittus' models yielded a relation of the same form as Holt's model, Eq. (3).

Twiss (1977) modelled the formation of subgrains, assuming that an initially uniform array of edge dislocations rearranges to form a minimum energy configuration, in an approach similar to that of Holt (1970) for cell formation. This minimization is achieved in the Twiss theory when the total energy of dislocations in a closed subgrain network is less than the total energy of the same dislocations distributed uniformly. This analysis leads to a relation- 
ship of the form of Eq. (1), but with $p$ a material independent parameter. Twiss (1977) then plotted a large amount of stress/subgrain size data (mainly for metals), and found that each individual set of data for a specific material obeyed Eq. (1), but with a spread of values of $p$ ranging from 1.62 for $\mathrm{Al}$ to 0.54 for austenitic stainless steel (AISI 316). However, the mean value of $p$ was found to be 1, and this was taken as evidence for the validity of Eq. (3).

Young and Sherby (1973) and Bird et al. (1969) also compiled a large amount of data from different materials, and both investigations found that Eq. (3) was a good average fit to the data. However, the spread of values of both $K$ and $p$ when Eq. (1) is fitted to the data for individual materials suggests that this equation is a better description of the data, and that $K$ and $p$ must be regarded as material-dependent parameters. Averaging data for a variety of materials is not justified.

In order to determine the possible dependence of the constants in Eq. (1) (or 3) on material or environmental parameters, it is necessary that an adequate model of the substructure forming process be formulated. The model should be dynamic, since the "average" substructure is a dynamic balance between a number of dislocation processes, and it should include the most important processes that contribute to that balance.

\section{A SIMPLE SUBGRAIN MODEL}

Consider a material undergoing steady state creep with a developed subgrain structure. This subgrain structure will be in a state of dynamic equilibrium with the free dislocations within the subgrains. As Takeuchi and Argon (1976) stated "... the steady substructure is only steady on a time average and is realized through a dynamic balance between the rate of formation of new boundaries by decomposition into cells of higher energy dislocation structures produced during an increase of strain and that of annihilation due to impingement of sub-boundaries of opposite sign moving in the opposite directions." In addition to this process, individual dislocations of both signs will impinge upon sub-boundaries (from both sides), contributing to the strain (rate). If the migrating sub-boundaries are regarded simply as groups of individual, mobile dislocations, the average subgrain boundary can be regarded as fixed, subject to incident dislocations of both signs. Additionally, annihilation due to sub-boundary motion is not the only process by which a steady state subgrain structure can be maintained. Even if the low angle boundaries are immobile, dislocations can climb within the boundary and annihilate with dislocations of opposite sign, as suggested by Blum (1971). If the rate of annihilation by this process can effectively keep the density of boundary dis- 
locations constant by cancelling out the effect of dislocations arriving at the boundary, a steady state substructure will be obtained.

Here, we will assume the average subgrain structure to be immobile, and analyze dislocation reactions at a typical boundary. However, the migration of boundaries is accounted for as the movement of individual component dislocations.

The rate of arrival of dislocations at any sub-boundary is determined by the rate of deformation within the subgrain, and also by the unimpeded slip distance in the material surrounding the sub-boundary, assuming that the sub-boundaries stop all dislocations which reach them. This unimpeded distance may be the distance to the next sub-boundary, but could be less if some other dislocation trapping process is occurring within the subgrain.

The rate of dislocation annihilation at the wall by boundary glide and annihilation is inversely dependent on the boundary separation, and the rate of annihilation due to dislocation climb within the boundary is inversely dependent on the wall length, which in turn is dependent on the separation. So, if boundaries start to form too close to each other, the supply of dislocations will not keep pace with the annihilation rate, and the boundaries will disband. Conversely, if boundaries form at a distance greater than some particular value, there is a greater likelihood that new sub-boundaries form in the spaces. In this way, a stable subgrain size will form depending on the equilibrium value of the misorientation rate.

In this section, a simple model of the subgrain structure and of the interaction of glide dislocations and the sub-boundaries is proposed, which leads to an expression connecting the subgrain size with stress. To simplify the calculations, we will assume the subgrains to be cubes, with simple tilt boundaries formed with dislocations of the same magnitude Burgers vector in an isotropic medium. Figure 1 schematically shows such an arrangement, with the boundaries in the plane of the page being pure twist. In the following we will ignore these twist boundaries where there will be no climb controlled dislocation annihilation, and adopt an essentially two dimensional model.

The dislocations arriving at any tilt wall will in general be of opposite signs, arriving from opposite sides of the wall under the action of the applied stress. However, an excess of dislocations of one sign (the same as those in the wall) must exist (geometrically necessary dislocations), due to local inhomogeneity of the deformation. If equal amounts of dislocations of both signs arrived at a wall they would climb toward each other and mutually annihilate, as in Weertman's (1975) model for climb controlled creep, and no sub-boundary would form. The boundaries form because the geometrically necessary excess of dislocations forms the low energy configuration. The density of these geometrically necessary dislocations is simply related to the local gradient of the strain (Ashby, 1970). In a polycrystal, for example, regions of inhomo- 


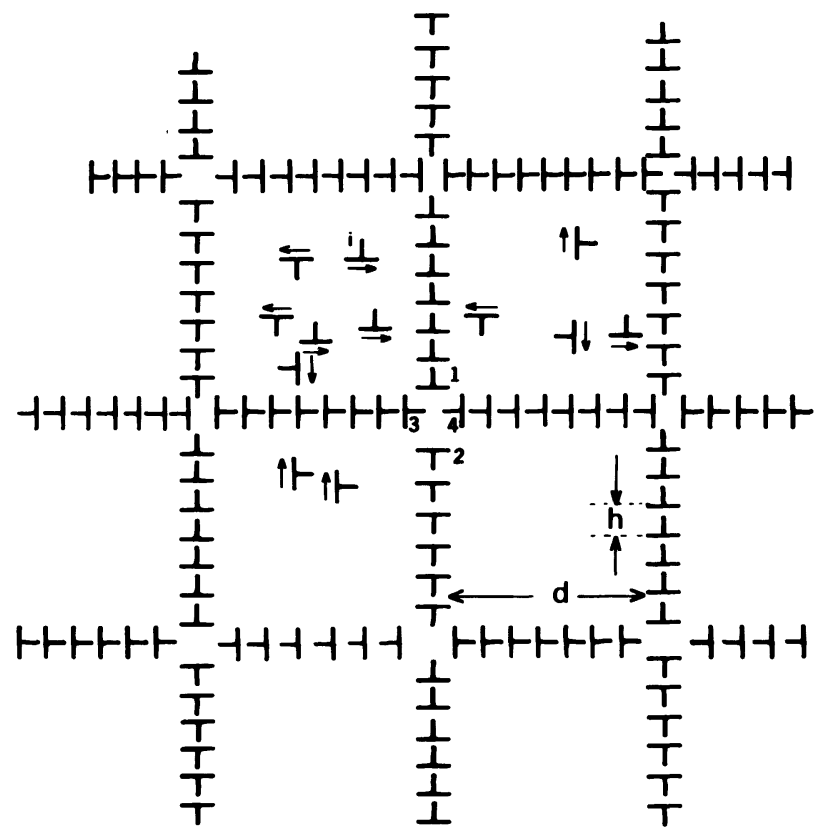

FIGURE 1 Idealized arrangement of dislocations to form a cubic subgrain boundary network. The subgrain size $(d)$ and the dislocation spacing $(h)$ are shown. Also shown are dislocations 1 to 4 at a typical boundary junction, and an incident dislocation (i).

geneous deformation and consequent strain gradients may tend to occur near grain boundaries.

If we define the following terms:

$L=$ total length of geometrically necessary dislocations in subgrain walls per unit volume ("smeared" wall density)

$\dot{L}^{+}=$rate of arrival of geometrically necessary dislocation length per unit volume at subgrain walls

and $\dot{L}^{-}=$rate of annihilation of geometrically necessary dislocation length per unit volume in subgrain walls,

then $\quad \dot{L}=\dot{L}^{+}-\dot{L}^{-}$

If the dislocations are separated by a distance $h$ in the walls of subgrains of size $d$, then assuming the tilt boundaries of Figure 1 are typical, we find:

$$
L=\frac{2}{d h}=\frac{2 \theta}{d b}
$$

where $\theta=b / h$ is the misorientation of the (small angle) tilt boundary and 
$b$ is the Burgers vector of the dislocations. Differentiating and allowing for changing misorientation but keeping the subgrain size constant, we obtain,

$$
\dot{L}=\frac{2 \dot{\theta}}{d b}
$$

Changing subgrain size can be allowed for here by including the appropriate $d$ term in Eq. (6); the contribution of the boundary movement to the strain rate must then be included in Eq. (7).

The dislocations arriving at the boundaries will comprise geometrically necessary dislocations which contribute to the misorientation and redundant dislocations which mutually annihilate. All dislocations will, however, contribute to the strain (rate), which can be considered to be composed of two contributions, that due to dislocation glide and climb within the subgrain, and that due to climb within the boundary. We will neglect the strain contribution from boundary climb, as we expect the number of redundant dislocations to be much larger than the number of geometrically necessary dislocations in most deformation conditions, and the redundant dislocations will have only a short distance to climb in order to annihilate, thus contributing little to the strain.

If $\dot{\rho}^{+}$represents the rate of (total) dislocation generation within the subgrain, and $l$ the mean slip distance, we can express the strain rate as (Blum, 1971)

$$
\dot{\varepsilon}=b l \dot{\rho}^{+}
$$

Only some of the dislocations created within a subgrain will contribute to the misorientation on reaching the wall. Here, we will assume that the proportion of these geometrically necessary dislocations is given by,

$$
\dot{L}^{+}=g \dot{\rho}^{+}
$$

where $g(o<g<1)$ is determined by the local deformation geometry, and is possibly stress dependent. At the moment we can only regard it as an empirical parameter, which for reasonably homogeneous strain fields will be a small number.

Substitution of Equation (8) in Equation (7) gives

$$
\dot{L}^{+}=\frac{g}{b l} \dot{\varepsilon}
$$

Assuming that the constitutive law for creep of the material is the familiar Dorn power law creep equation, i.e.

$$
\dot{\varepsilon}=A \frac{D_{1} G b}{k T}\left(\frac{\sigma}{G}\right)^{n}
$$


then

$$
\dot{L}^{+}=\frac{g}{b l} A \frac{D_{1} G b}{k T}\left(\frac{\sigma}{G}\right)^{n}
$$

where $\quad D_{1}=$ coefficient of diffusion for creep

$G=$ shear modulus

$k=$ Boltzmann's constant

$T=$ temperature

and $A, n$ are dimensionless material constants.

It now remains to calculate $\dot{L}^{-}$, the rate of annihilation of the geometrically necessary dislocations in the boundary. It is tacitly assumed that the redundant dislocations annihilate more rapidly on average, as in general they will only have to climb short distances along the wall before encountering a dislocation of opposite sign.

Consider a boundary junction such as that in the centre of Figure 1. We will examine dislocation reactions within one wall and one junction only as this is typical of the entire subgrain array in Figure 1. In isolation, the two parallel opposite edge dislocations 1 and 2 will experience a mutually attractive climb force, as will dislocations 3 and 4 . This means that there will be a tendency for the dislocation quadrupole at the boundary junction to contract, and for the two pairs of opposite edge dislocations to mutually annihilate. It is the balance between this annihilation and the input of dislocations from the deforming subgrain which we consider in the following.

We can ignore the different mobilities of dislocations climbing "up" by interstitial emission (dislocations 3 and 4) and dislocations climbing "down" by vacancy emission (dislocations 1 and 2) (see Nabarro, 1967, p. 351), because the short path for vacancy flux between the "up" and "down" dislocations in the region of the boundary junction will reduce any differences in velocity.

If we know $t_{c}$, the average time taken for a geometrically necessary dislocation to climb to the sink at the end of the wall (Figure 2), $\dot{L}^{-}$can be calculated from

$$
\dot{L}^{-}=2 L / t_{c}
$$

Figure 2a shows the dislocation wall of Figure 1 upon which glide dislocations are impinging. In general they would have to climb a little $($ by $<h / 2)$ to be able to fit into the wall between two of the wall dislocations (Amelinckx and Strumane, 1960). $t_{c}$ will be given by the time taken by a typical incident dislocation ( $i$ in Figure 2 ) to climb to the sink at the boundary junction. To calculate this, we would have to consider the train of dislocations "shuffling" down under the influence of all the other wall dislocations. Such a detailed approach is not warranted. In order to estimate $t_{c}$, we will consider the simple problem of the approach by climb of the two opposite sign edge dislocations 2 and $i$ (see Figure 2), regarding dislocation 2 as fixed. For the moment we 


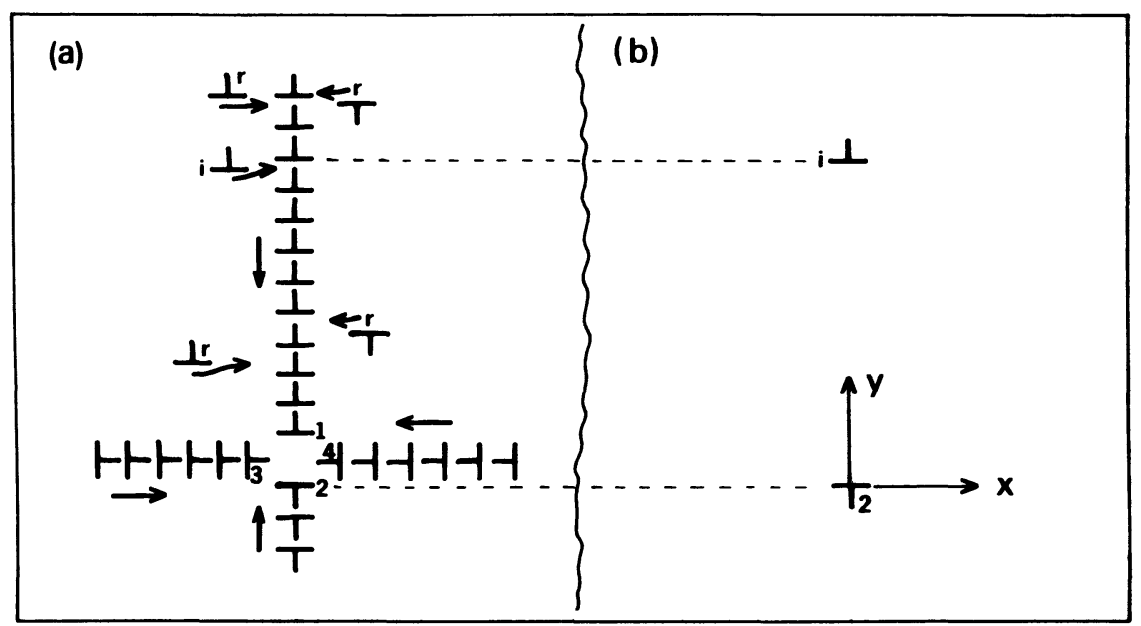

\section{FIGURE 2}

a) A section of boundary showing a junction which acts as a sink for dislocations such as dislocation $i$ which impinge on the boundary and climb down to the junction. Also shown are two pairs of redundant dislocations (labelled $r$ ) which mutually annihilate in pairs.

b) Simplified picture of Figure 2(a) used in the text to consider the climb of dislocation $i$ to the junction at dislocation 2. Dislocation 2 is regarded as fixed and as supplying the climb force acting on dislocation $i$, which has joined the boundary.

ignore the presence of the other dislocations in the walls, as the stress due to dislocations above and below dislocation $i$ will tend to cancel. Taking the origin of coordinates at dislocation 2 , the climb velocity of dislocation $i$ will be given by (Nabarro, 1967)

$$
v_{c}(y)=\frac{D_{2} b}{k T} b c_{j} F_{y}
$$

$c_{j}=$ number of jogs per unit dislocation length

$F_{y}=$ driving force per unit dislocation length

and $D_{2}=$ appropriate diffusion coefficient for dislocation climb in the boundary.

The effect of the other wall dislocations must now be considered, however. Eq. (13) gives the climb velocity for dislocation $i$ in isolation (except for dislocation 2), when a jog is able to emit vacancies into a (roughly) spherical volume surrounding it. The presence of the neighbouring dislocation changes this volume into a flat pill box shape, height $h$ (the separation of the wall dislocations) (see Figure 3). This effectively limits the vacancy flux from the dislocation, and will retard its climb rate by a factor of about $h / d \sim b / \theta d$. That is, the climb velocity will be more accurately given by multiplying the velocity given by Eq. (13) by $b / \theta d$, giving: 
(a)

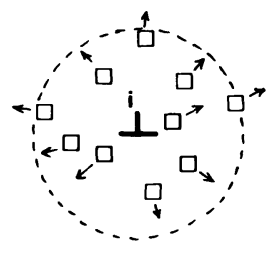

(b)

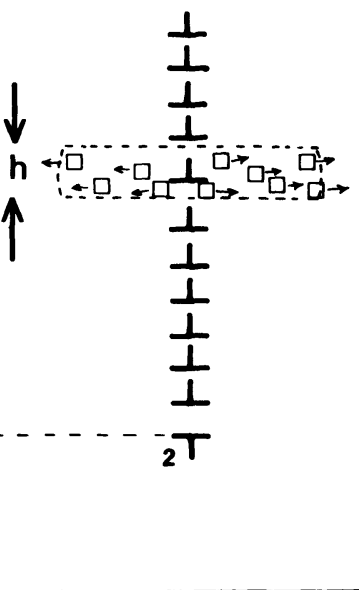

\section{FIGURE 3}

a) Illustration of dislocation $i$ climbing down toward dislocation 2 by the emission of vacancies (shown as squares). The vacancies can be emitted by each jog on dislocation $i$ into a roughly spherical volume in the absence of the other wall dislocations.

b) Illustration of dislocation $i$ climbing towards dislocation 2 in the presence of the other wall dislocations, which are also climbing in the same direction. Because they also are emitting vacancies, they will effectively restrict the volume into which a jog on $i$ can emit vacancies to a pill box shape, thickness $h$, so retarding the climb rate.

$$
v_{c}(y)=\frac{1}{\theta} \frac{D_{2} b}{k T} b c_{j} \frac{b}{d} F_{y}
$$

The driving force for the climb of dislocation $i$ will be that due to dislocation 2. We are ignoring the climb force due to the imposed stress, which is the same for all dislocations of the same type. The force on dislocation $i$ will be given by (Hirth and Lothe, 1968, p. 513):

$$
F_{y}=-\frac{G b}{2 \pi(1-v)} \cdot \frac{1}{y}
$$

where $v$ is the Poisson's ratio.

Substitution of Eq. (15) in Eq. (14) gives,

$$
v_{c}(y)=-\frac{1}{\theta} \frac{D_{2} G b}{k T} b c_{j} \frac{b}{d 2 \pi(1-v)} \cdot \frac{b^{2}}{y}
$$

Assuming the average climb distance to be $d / 4, t_{c}$ will be given by

$$
t_{c}=\int_{d / 4}^{0} \frac{1}{v_{c}(y)} d y
$$


Integration and substitution in Eq. (12) gives

$$
\dot{L}^{-}=\frac{1}{b d} \frac{D_{2} G b}{k T} b c_{j} \frac{64}{\pi(1-v)}\left(\frac{b}{d}\right)^{3}
$$

Substitution of Eq. (18), (11) and (6) in (4) gives, on manipulation,

$$
g A\left(\frac{\sigma}{G}\right)^{n}=\frac{2 l}{d}\left[\frac{32}{\pi(1-v)} \frac{D_{2}}{D_{1}} b c_{j}\left(\frac{b}{d}\right)^{3}+\frac{k T}{D_{1} G b} \theta\right]
$$

\section{APPLICATION TO STEADY STATE SUBSTRUCTURES}

In metals where a subgrain structure develops during creep, it is generally found that the average subgrain size and boundary misorientation do not increase with strain, but are in dynamic equilibrium, where subgrains are forming and disbanding constantly (Takeuchi and Argon, 1976). The only data to the contrary concerns aluminium (Exell and Warrington, 1972; Orlovà et al., 1972) and this is discussed in the following section.

If, in Eq. (19) we assume that the misorientation is constant $(\theta=0)$, then the expression relating stress and subgrain size becomes

$$
\frac{\sigma}{G}=\left[\frac{64}{\pi g A(1-v)} \frac{D_{2}}{D_{1}} b c_{j}\right]^{\frac{1}{n}}\left(\frac{l}{d}\right)^{\frac{1}{n}}\left(\frac{b}{d}\right)^{\frac{3}{n}}
$$

Comparison of Eq. (20) with the empirical Eq. (1) shows that they are the same if the empirical constants $K$ and $p$ are defined by

$$
K=\left[\frac{64}{\pi g A(1-v)} \frac{D_{2}}{D_{1}} b c_{j} \frac{l}{b}\right]^{\frac{1}{n}}
$$

and

$$
p=4 / n
$$

However, $K$ and $p$ are not universal constants, but are dependent on the creep properties of the material (or the details of the creep mechanism), and $K$ also has an explicit temperature dependence contained principally in the terms $D_{1}, D_{2}$ and $c_{j}$.

\section{Temperature dependence}

The relation between stress and subgrain size given by Eq. (20) will be altered implicitly by the temperature dependence of $G$ (the shear modulus), any variation in the creep constants $\boldsymbol{A}$ and $\boldsymbol{n}$ due to changes in creep mechanism, and possibly variations in $g$. However, if the creep constitutive relation given 
by Eq. (10) is accurate over the temperature range of interest, such variations can be ignored.

Explicit temperature dependence, however, enters through the jog concentration term and the two diffusion coefficients. Writing

$$
b c_{j}=\exp \left(-U_{j} / k T\right)
$$

where $U_{j}$ is the jog formation energy,

$$
D_{1}=D_{01} \exp \left(-Q_{1} / k T\right)
$$

and

$$
D_{2}=D_{02} \exp \left(-Q_{2} / k T\right)
$$

where $Q_{1}$ and $Q_{2}$ are the activation energies for the two diffusion processes, and $D_{01}$ and $D_{02}$ the diffusion pre-exponentials, the temperature dependence of $K$ in Eq. (21) can be written as

Here,

$$
K(T)=K_{o} \exp \left(-Q_{K} / k T\right)
$$

and

$$
K_{o}=\left[\frac{64}{\pi g A(1-v)} \frac{l}{b} \frac{D_{02}}{D_{01}}\right]^{\frac{1}{n}}
$$

Data for the jog formation energies are rare, but an estimate for $U_{j} \simeq 0.2 \mathrm{eV}$ has been given by Friedel $(1964$, p. 309$)$. This value (equivalent to about $20 \mathrm{~kJ} / \mathrm{mole}$ ) is an order of magnitude less than the values expected for $Q_{2}$ and $Q_{1}$. In addition, if the diffusion process controlling the creep is lattice vacancy diffusion (activation energy $Q_{1}$ ), and if $Q_{2}$ is the activation energy for dislocation core diffusion, we expect that $Q_{1}-Q_{2} \gtrless 0$, and this would appear to hold generally. Subtracting $Q_{1}-Q_{2}$ from the small quantity $U_{j}$ and then dividing by $n \geq 3$ leads to an extremely small apparent activation energy $Q_{K}$, and a consequent weak temperature dependence of $K$ in Eq. (21). This dependence can be of either sign, depending on the sign of $Q_{K}$. If the climb processes controlling creep and climb within the sub-boundary have the same activation energy, there would be virtually no explicit temperature dependence of $K$. This would occur in particular if sub-boundary pile ups were the rate controlling step for creep, but this need not be the case.

\section{Dependence on creep mechanism}

Eq. (20) predicts that the exponent in the stress/subgrain relationship will depend on the creep exponent, and hence on the mechanism of creep. In addition, the relationship of slip distance $l$ to the subgrain size $d$ will alter 
the exponent (and the pre-exponent). There are three different possibilities here.

1) Slip limited by sub-boundaries - If all dislocations created within the subgrains can be assumed to reach the sub-boundary where annihilation with dislocations of opposite sign occurs, the slip distance will be given by

$$
l=d / 2
$$

Eq. (20) then gives

$$
\frac{\sigma}{G}=\left[\frac{32}{\pi g A(1-v)} \frac{D_{2}}{D_{1}} b c_{j}\right]^{\frac{1}{n}}\left(\frac{b}{d}\right)^{\frac{3}{n}}
$$

A log-log plot of stress as a function of subgrain size should, if this relationship holds, have a gradient of $-3 / n$.

2) Slip distance constant-If the bulk of the dislocations within the subgrain can be regarded as slipping a fixed distance $\bar{l}$ before annihilating or becoming permanently immobilized, then

$$
l=\bar{l}
$$

Substitution in Eq. (20) gives

$$
\frac{\sigma}{G}=\left[\frac{64}{\pi g A(1-v)} \frac{\bar{l}}{b} \frac{D_{2}}{D_{1}} b c_{j}\right]^{\frac{1}{n}}\left(\frac{b}{d}\right)^{\frac{1}{n}}
$$

This does not seem a particularly physically plausible assumption, but Evans and Knowles (1977), in testing a network creep model, found that in many cases this assumption fitted data more closely than equating the slip distance with the network size. This relationship predicts a gradient of $-4 / n$ for a $\log -\log$ plot of stress as a function of subgrain size.

3) Slip distance controlled by network-If the creep process is occurring within a three-dimensional network (Friedel network), and the slip distance is limited by the size of this network $\bar{x}$, we have

$$
l=\bar{x}
$$

If $\bar{x}$ is inversely proportional to the stress, as is theoretically predicted, we can write, following Evans and Knowles (1977),

$$
\frac{\bar{x}}{b}=\alpha \frac{G}{\sigma}
$$

where $\alpha$ is a constant of order 1 . 
Substitution of Eq. (33) and (34) in (20) gives,

$$
\frac{\sigma}{G}=\left[\frac{64 \alpha}{\pi g A(1-v)} \cdot \frac{D_{2}}{D_{1}} b c_{j}\right]^{\frac{1}{n+1}}\left(\frac{b}{d}\right)^{\frac{4}{n+1}}
$$

In this case a gradient of $-4 /(n+1)$ is predicted for a plot of stress as a function of subgrain size.

\section{SIGNIFICANCE OF SUBGRAIN ROTATION}

In minerals, it cannot be assumed that the rate of misorientation increase of subgrain boundaries is very small. The phenomenon of "subgrain rotation" was first observed in quartz by Hobbs (1968), and has since been widely documented for many minerals (White, 1973; Poirier and Nicolas, 1975; Bell and Etheridge, 1976; Guillope and Poirier, 1979; Etheridge and Kirby, 1982) as an important mechanism of dynamic recrystallization. If we assume as a preliminary simplification that the degree of misorientation is linearly dependent on the strain, then we have

$$
\dot{\theta}=\varphi \dot{\varepsilon}
$$

where $\varphi$ is a constant. The misorientation in aluminium has been found to obey Eq. (36) by Orlovà et al. (1972), with $\varphi \simeq 0.02$. Exell and Warrington (1972) also found a monotonic increase in subgrain misorientation with strain in aluminium, but the rapid increase during the first $1 \%$ strain was followed by a much slower misorientation increase. In this case Eq. (36) would need some modification. In halite, where rotation recrystallization was observed, Guillope and Poirier (1979) found that after about $60 \%$ shortening misorientations were commonly of the order of $30^{\circ}$, implying a large value of $\varphi$ if Eq. (36) is assumed to hold. To estimate $\varphi$ average misorientation values would be needed, however. Etheridge and Kirby (1982) also found very rapid rotations of subgrains and consequent recrystallized grains in orthopyroxene.

Substitution of Eq. (36) in Eq. (19) and using the creep constitutive law (Eq. 10) gives:

$$
\frac{\sigma}{G}=\left[\frac{64}{\pi A(1-v)} \cdot \frac{D_{2}}{D_{1}} b c_{j}\right]^{\frac{1}{n}}\left[\frac{l / d}{g-3 \varphi l / d}\right]^{\frac{1}{n}}\left(\frac{b}{d}\right)^{\frac{3}{n}}
$$

The three different substitutions for the slip distance $l$ can be made, as in the previous section, resulting in stress-subgrain relationships of a slightly more complicated mathematical form than those obtained for constant misorientation (Eq. 30, 32 and 35). The procedure is straight forward, and will 
not be given here, as the effects of allowing for misorientation increase can be seen from Eq. (37). The increasing misorientation changes the constant ( $K$ in Eq. (1)) in the stress-subgrain size relation, and also could have an effect on the exponent, due to the appearance of $d$ in the denominator of the second term on the right hand side of Eq. (37). However, experimental testing of these effects is probably not feasible, as the rate of misorientation would have to be altered, keeping all other microstructural parameters constant.

\section{COMPARISON WITH EXPERIMENT}

In order to compare the predictions of Eq. (19) with available experimental data, we will make the following simplifying assumptions.

i) We will ignore any misorientation changes $(\dot{\theta}=0)$. This is reasonable for metals (except perhaps aluminium), and affects the conclusions little with regard to mineral data.

ii) The same diffusion process controls both the creep described by Eq. (10) and the sub-boundary climb and annihilation (Eq. (18)). That is, $D_{1}=D_{2}$.

iii) $U_{j}=0.2 \mathrm{eV}$ (Friedel, 1964). Substitution in Eq. (23) gives $b c_{j}=0.1$

iv) $v=0.3$ (roughly true for all materials considered).

With these assumptions, Eq. (19) simplifies to:

$$
\frac{\sigma}{G}=\left[\frac{2.91}{g A}\right]^{\frac{1}{n}}\left(\frac{l}{d}\right)^{\frac{1}{n}}\left(\frac{b}{d}\right)^{\frac{3}{n}}
$$

Regarding $\sigma / G$ and $d / b$ as the dependent and independent variables respectively, the relationship involves four parameters: $g, A, l$, and $n$.

The slip distance $l$ can be interpreted as $d / 2, \bar{l}$ or $\bar{x}$, resulting in exponents of $b / d$ ( $p$ in Eq. (1)) of $3 / n, 4 / n$ and $4 / n+1$ respectively.

For $n>3,3 / n<4 /(n+1)<4 / n$, and so we will consider only the equations appropriate to $l=d / 2$ and $l=l$, the gradient of the $l=\bar{x}$ equation being intermediate to these for $n>3$, which holds for most materials under conditions at which they form subgrains.

Assuming $l=d / 2$, Eq. (38) becomes:

$$
\frac{\sigma}{G}=\left(\frac{1.5}{g A}\right)^{\frac{1}{n}}\left(\frac{b}{d}\right)^{\frac{3}{n}}
$$



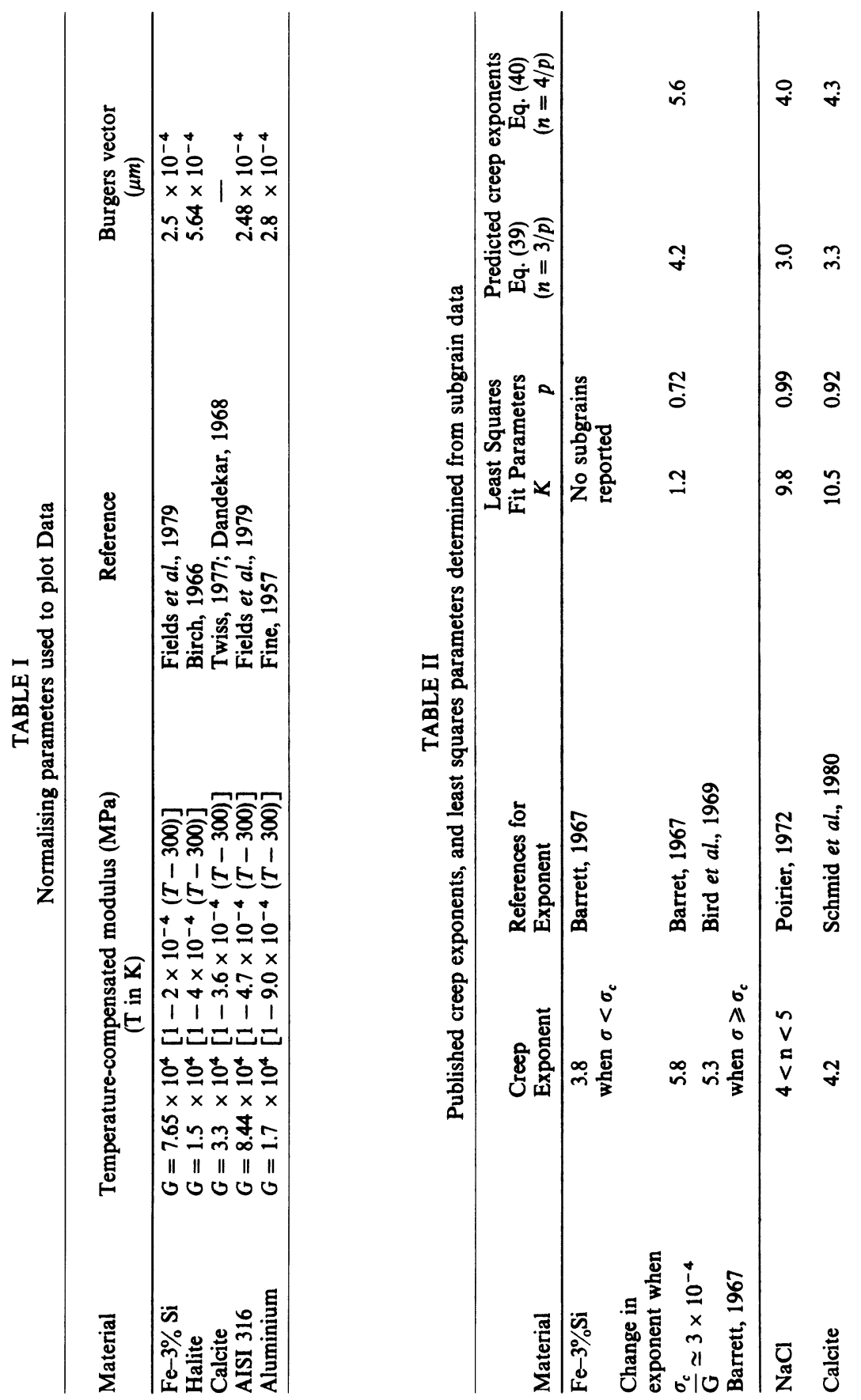


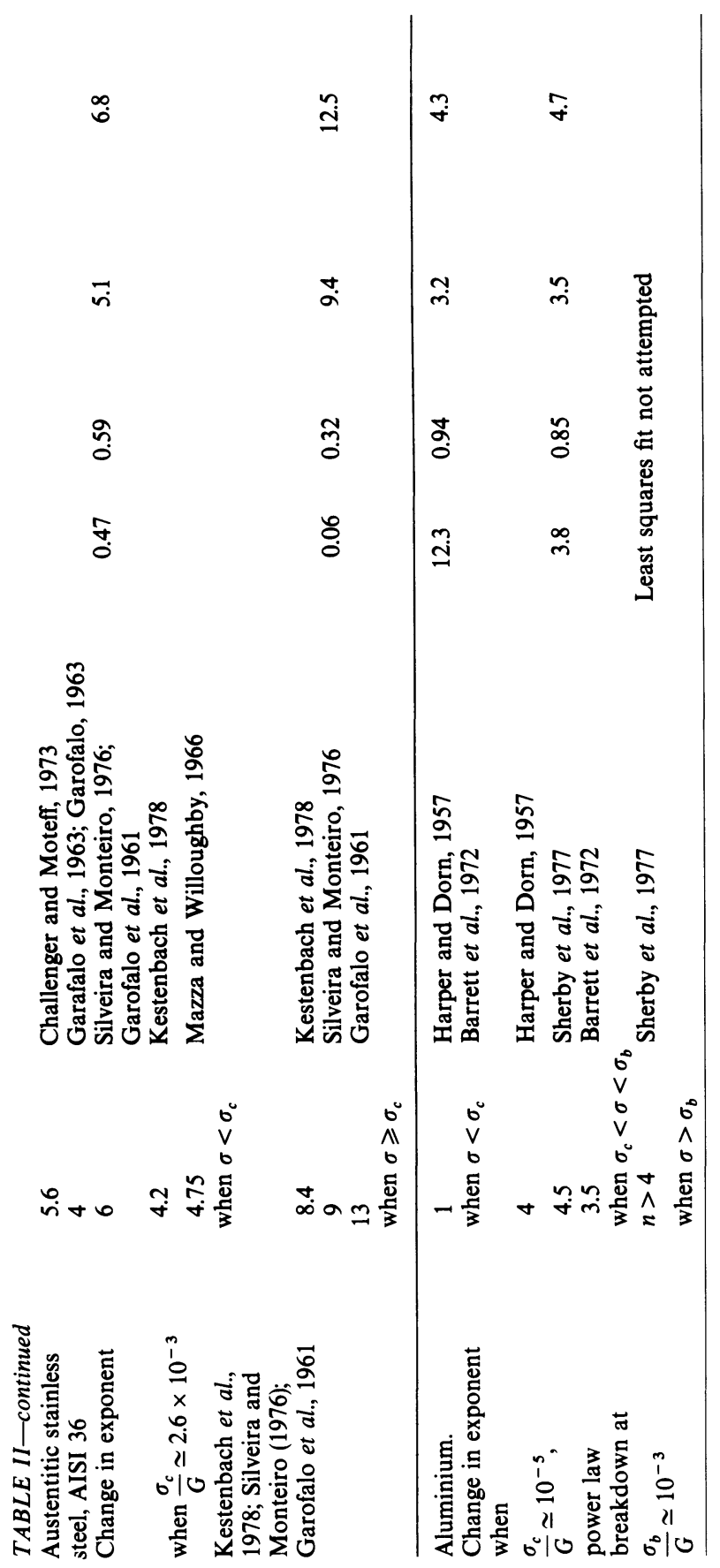


Evans and Knowles (1977) assume that the fixed slip distance in their model is $2 \mu \mathrm{m}$. In approximate accord with this we will assume that $\bar{l}=10^{3} \mathrm{~b}$, and so substituting $l=\bar{l}$ in Eq. (38) we get

$$
\frac{\sigma}{G}=\left(\frac{2910}{g A}\right)^{\frac{1}{n}}\left(\frac{b}{d}\right)^{\frac{4}{n}}
$$

Eq. (39) and (40) are now capable of predicting the stress as a function of subgrain size for a particular material; this relationship involves the appropriate creep exponent $n$ and the constants $A$ and $g$. To check the validity of these two equations we have collected published values of stress as a function of grain size for a number of materials, including all data where the subgrain size has been determined by electron microscopy involving large numbers of subgrains, by etch pitting methods, or by optical thin section (in the calcite). Examination of data makes it evident that X-ray methods overestimate subgrain size, and so X-ray determinations have been ignored. The data were normalized using the parameters given in Table I. A least squares fit of Eq. (1) to the set of data for each individual material was made, this being a linear relationship on a log-log plot. In cases where the creep exponent $n$ is known to change with stress the linear least squares fit was made to points within the range of stress values where $n$ can be regarded as constant. The published values of the creep exponent are given for each material in Table II, along with the quantities $p$ and $K$ determined from the least squares fitting procedure. The constant $K$ includes the parameters $g$, $A$, and $n$ in Eq. (39) and (40). Comparison of Eq. (39) of Eq. (40) with Eq. (1) shows that the value of $n$ in Eq. (39) or (40) needed to fit the data can be simply calculated from $p$, the negative gradient of the graph. The values of $n$ so determined are given in Table II. In principle, the value of $g$ necessary to fit the data can also be calculated but this requires a value for $A$, and this is, in general, poorly known, and so no estimate of $g$ has been given. For the "high $n$ " material $\mathrm{Fe}-3 \% \mathrm{Si}$ and the two $n \sim 4$ materials $\mathrm{NaCl}$ and calcite the gradient of the stress/subgrain size plot certainly appears to correlate with the value of $n$ for the particular material. In the case of the austenitic stainless steel, the gradient of the stress/subgrain size plot changes at about the same stress level at which the creep exponent is observed to change, giving further support to the proposal that the gradient $p$ in Eq. (1) is inversely dependent on the creep exponent. For aluminium in the intermediate stress regime the correlation is reasonable, but in the high- and lowstress regimes it is not good. In the high stress region the bulk of the data are from the high strain rate extrusion experiments of McQueen et al. (1967) and any effects of stress gradients in this highly non-linear material are not 


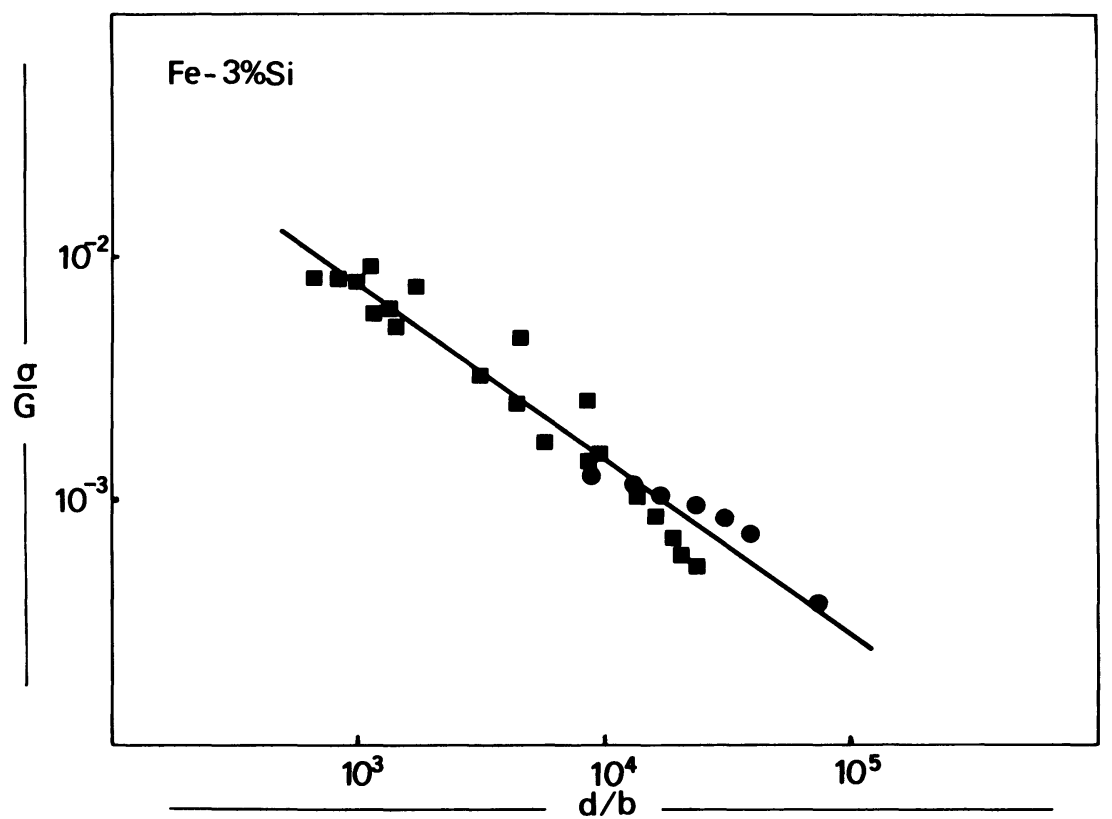

FIGURE 4 A least squares fit (solid line) to stress/subgrain size data for $\mathrm{Fe}-3 \% \mathrm{Si}$ alloy. Data from Young and Sherby (1973)(squares) and Barrett, Nix and Sherby (1966)(circles). All subgrains measured used TEM. See Table II for least squares fit parameters.

accounted for. In the low stress $(n \simeq 1)$ region, the large spread of the data makes the least squares fitting procedure unreliable.

Overall, the evidence supports the proposal that the gradient of the stress/ subgrain plot is not necessarily -1 , but in fact is a material dependent property inversely related to the creep exponent.

In Figures 4, 5 and 6 the three new data compilations and the resultant fitted lines are given. The data for halite subgrains of Guillope and Poirier (1979) and calcite subgrains of Schmid et al. (1980) have not been plotted, as, in both cases, all the data came from the one source.

\section{DISCUSSION}

\section{Comparison with creep models}

Generally, models for creep commence by deriving a relationship between strain rate and some structural parameter such as network spacing (Evans and Knowles, 1977), subgrain size (Weertman, 1968), slipband spacing 


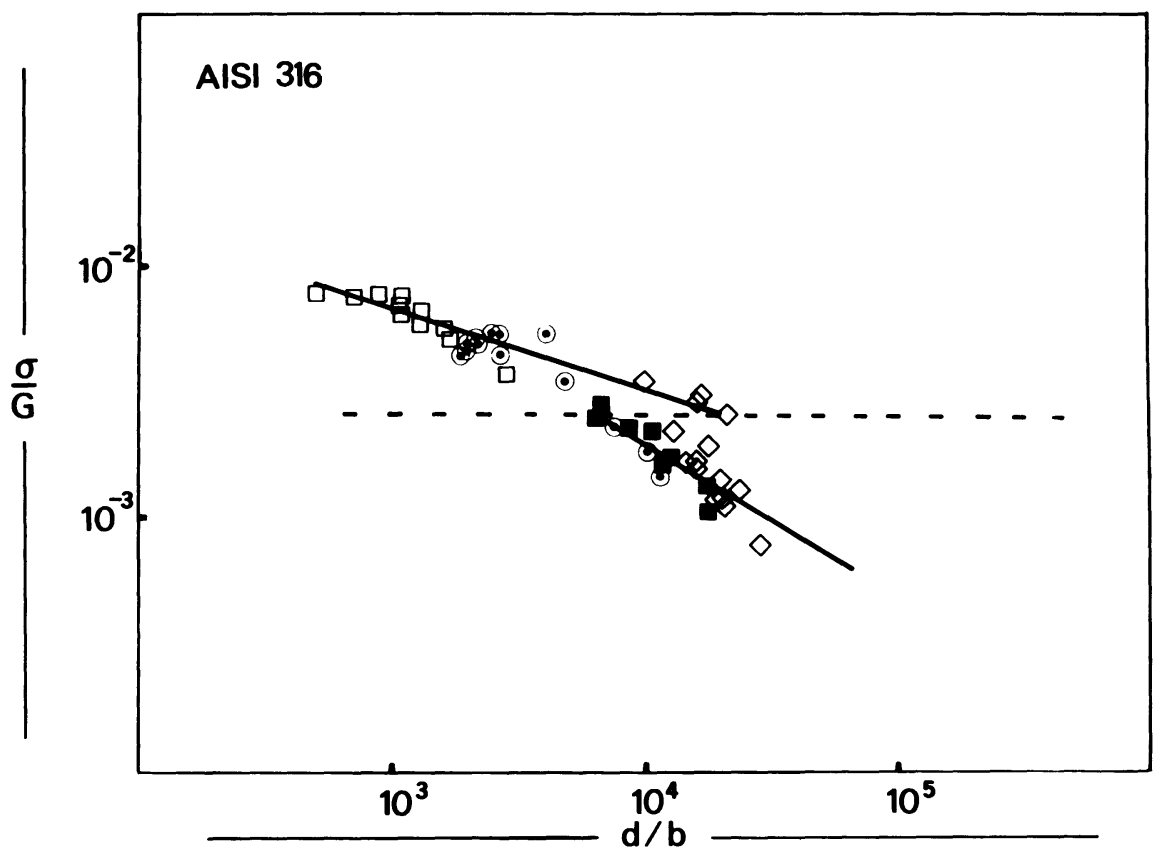

FIGURE 5 Two least square fits (solid lines) to stress/subgrain size data for austenitic stainless steel, AISI 316. Each fit was, in turn, to data exclusively above or below the broken line, which represents the stress at which the creep exponent changes (see Table II). No attempt was made to couple the least squares fitting procedure to maintain continuity at the changeover stress. Data from Young and Sherby (1973) (open squares); Michel et al. (1973) (centred open circles); Challenger and Moteff (1973) (shaded squares); and Garofalo et al. (1963) (open diamonds), all measurements being from TEM. See Table II for least squares fit parameters.

(Spingarn and Nix, 1979), or dislocation spacing. Elimination of this structural term by empirically relating it to the stress results in the constitutive equation (usually a power law). This equation is then tested by matching its predictions with experiments, the crucial test being the matching of exponents. Good agreement is rarely obtained, and no theory of creep at this stage can completely explain the variations in the values of $n$ displayed by many materials.

We have chosen here to regard $n$ as a material property, and to use the empirical constitutive equation for the material. By modelling the connection between strain rate and the subgrain size, we have thus obtained the stress/ subgrain size relationship given as Eq. (19). The appearance of $n$ in this relationship does not imply that the subgrain network is controlling the rate of deformation, but rather that the processes influencing the steady state creep rate also influence the subgrain structure. The interaction of dislocations with the subgrain boundaries could be the rate controlling step, but it is not a 


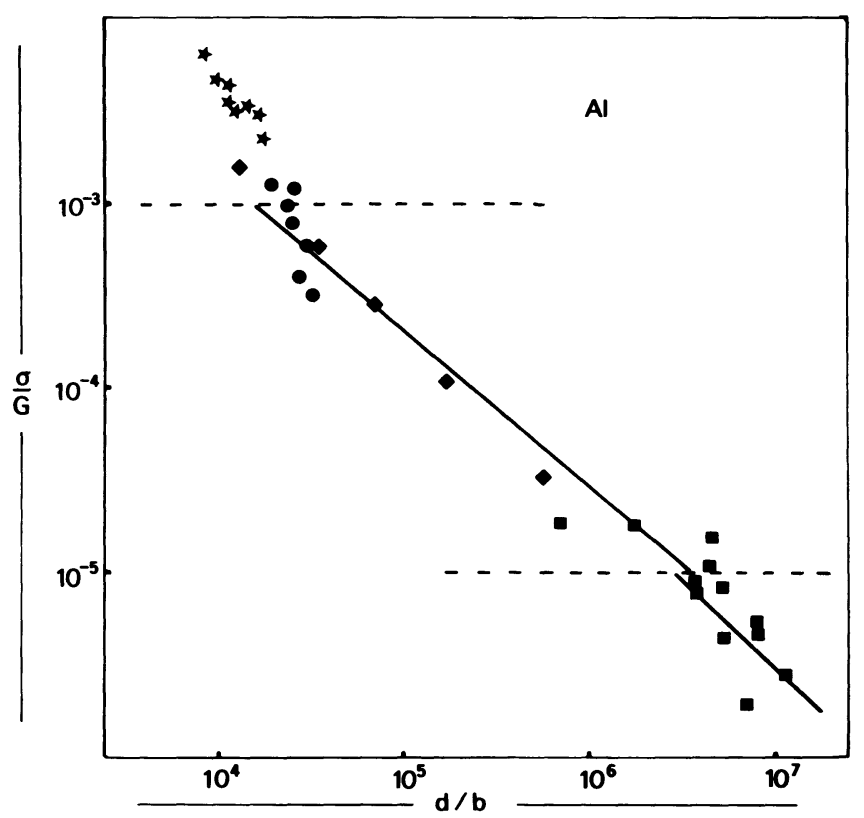

FIGURE 6 Two least squares fits (solid lines) to stress/subgrain size data for aluminium. Each fit was, in turn, to data exclusively above or below the lower broken line, which represents a stress at which the creep exponent changes (see Table II). No attempt was made to couple the least squares fitting procedure to maintain continuity at this changeover stress, and no least squares fit was attempted to data above the upper broken line, (the region of "power law breakdown," see Table II). Data from McQueen et al. (1967) (stars); Sherby et al. (1977) (diamonds); Orlovà et al. (1972) (circles); and Barrett et al. (1972) (squares). See Table II for least squares fit parameters.

necessary assumption of the model that this be so. The model for stress/ subgrain size relationship is independent of the arguments of Robinson and Sherby (1969) who postulate a direct dependence of creep rate on subgrain size, and of the contrary view taken by Parker and Wilshire (1976) who explain the results of the same stress drop tests in terms of limited recovery.

\section{The fixed slip distance}

In the comparison between published experimental data and the predictions of the theory, the correlation between the creep exponent $n$ and the gradient of the stress-subgrain size plot is best represented by Eq. (40), which predicts a $-4 / n$ exponent in the stress-subgrain size relationship. The alternative predictions of gradients of $-3 / n$ or $-4 /(n+1)$ (which in fact are quite close to each other over the usual range of $n$ values) are not satisfactory. That 
is, for the proposed model the assumption that the mean slip distance is constant leads to the better explanation of the results. Attaching any physical reality to this implies acceptance of the other physical features of the model which lead to the prediction of this exponent, but the finding of a fixed slip distance is not unprecedented. Evans and Knowles (1977) in testing their dislocation network creep model used two values for the slip distances-a fixed slip distance and the network spacing. They found that the fixed slip distance usually gave better agreement with experiments, as we did here, but they accounted for the fixed distance by equating it with dislocation barriers due to "extraneous features such as sub-boundaries, etc." This cannot really be so, because if sub-boundary spacing is inversely dependent on stress (if not precisely linearly inverse) it is a variable like the network spacing, and not surprisingly in this paper the results of assuming the slip distance to be network- or sub-boundary-limited are similar to each other, whereas the fixed slip distance assumption leads to a distinctly different result.

\section{The use of subgrains as palaeopiezometers in rocks and minerals}

Subgrain sizes in naturally deformed minerals have relatively restricted ranges (e.g. $\sim 1$ order of magnitude for quartz), which predict that crustal stresses range between about 10 and $150 \mathrm{MPa}$ using the equations of Twiss (1977). This range of stress is within the limits imposed by other techniques (Hanks, 1977; McGarr and Gay, 1979), but it does not necessarily follow that the stress calculated from a single sample or even a suite of samples is accurate to better than an order of magnitude. In fact, the differences between empirical relations from a number of workers (e.g. Twiss, 1977; Mercier et al., 1977; Christie et al., 1980; Ord, 1981; for quartz); and some of the dislocation density and subgrain and recrystallized grainsize data from naturally deformed rocks (White, 1979a; Etheridge and Wilkie, 1981; Weathers et al., 1980) suggest that environmental and/or material variables may give rise to significant, stressindependent variations in grainsize. In this context, it is useful to examine the likely magnitude of the environmental and material parameters in Eq. (19) with particular reference to minerals.

The major influence on the stress/subgrain size relationship will be via the thermally activated terms $D_{1}, D_{2}$ and $c_{j}$. The temperature-dependence arising from these terms has been discussed in general terms above, and it was concluded that it would be small for metals and alloys. However, in minerals (and other insulators and semi-conductors) there is the added complexity that the jogs may be charged (Hirsch, 1981; Hobbs, 1981, 1982) adding an additional energy term to $U_{j}$ in Eq. (23), namely, the difference between the Fermi energy and the ionization energy of the jog. In insulators this could be of the order of $1 \mathrm{eV}$, so that the charged jog concentration may be more 
temperature-dependent than in metals, leading in turn to a stronger temperature-dependence of the stress/subgrain size relation in minerals.

Moreover, in non-metals, the terms $D_{1}, D_{2}$ and $c_{j}$ also vary with the defect chemistry of the material (Hirsch, 1981; Hobbs, 1981) giving rise to a complex interaction between temperature and defect chemistry dependences. This is especially true for $c_{j}$ which is expected to be particularly sensitive to the position of the Fermi level. The best documented example of the relationship between defect chemistry and creep properties in minerals comes from the most recent experiments on olivine (Gueguen, 1979; Hornack and Kohlstedt, 1979; Ricoult and Kohlstedt, 1980; Jaoul et al., 1981). The variations of the creep diffusion parameter $\left(D_{1}\right)$ for olivine with the thermodynamic actitivies of $\mathrm{O}_{2}, \mathrm{Mg}$ and $\mathrm{Si}$ are consistent with the rate controlling step being the migration of positively charged jogs by a silicon vacancy diffusion mechanism (Hobbs, 1982). If this is so, the sub-boundary climb process will be similarly, although not necessarily identically, affected, and the stress/subgrain size relation will depend on geochemical environment. Published stress/subgrain size equations for minerals have all been determined from experiments whose chemical environment is poorly constrained, and so it is not possible to test this chemical dependence quantitatively from the published data. However, the older data for olivine provides some qualitative verification. Ross et al. $(1980$, Figures 12, 13) have summarized the bulk of the available stress/ subgrain size and stress/recrystallized grainsize data for olivine. First, there are significant differences between their own "wet" and "dry" experimentally determined relations for both subgrains and recrystallized grains. The specific chemical environments of their "wet" and "dry" experiments are unknown, but they must differ significantly in $f_{\mathrm{H}_{2} \mathrm{O}}$ (and thus $f_{\mathrm{H}_{2}}, f_{\mathrm{O}_{2}}$ ). In addition, the fact that the $\mathrm{H}_{2} \mathrm{O}$ in "wet" experiments is derived from the breakdown of talc to forsterite may give rise to differences in the thermodynamic activities of $\mathrm{Mg}$ and $\mathrm{Si}$. Second, their "dry" stress/subgrain size equation for Mt. Burnett dunite differs markedly from the equation determined by Mercier et al. (1976). The origin of the latter material is not entirely clear, but it is presumably the unidentified lherzolite deformed by Raleigh and Kirby (1970). The presence of both orthopyroxene and diopside in the lherzolite would have given rise to different thermodynamic activities of $\mathrm{Mg}, \mathrm{Si}, \mathrm{Ca}$ and $\mathrm{Fe}$ to those in the dunite, both during original crystallization and during experimental deformation. As discussed by Hobbs (1982), these differences may lead to substantial differences in the thermally activated creep parameters, and thus the stress/subgrain size equation.

Other variables in Eq. (19) may have a small influence on stress/subgrain size relationship, in particular $g$ and $\dot{\theta}$. As stated above (p. 7), $g$ will generally be small, but it may be higher in minerals than in metals because minerals have more restricted slip geometries, and are thus more prone to hetero- 
geneous straining (i.e. deformation bands, kinks, core and mantle structure). Sub-boundary misorientation, $\theta$, commonly increases with strain in minerals, leading to the phenomenon of subgrain rotation recrystallization (Poirier and Nicolas, 1965; Bell and Etheridge, 1976; White, 1977). Eq. (19) predicts that subgrain size will increase with both increasing $\theta$ (via the climb velocity in the boundary) and $\dot{\theta}$, and this broadly consistent with observations on naturally recrystallized materials (Bell and Etheridge, 1976; White, 1979a).

\section{CONCLUSIONS}

A dynamic model of subgrain formation during dislocation creep has been formulated, giving rise to a stress/subgrain size relationship that reflects the details of the creep process and includes all variables that affect this process. The most important of these variables are the creep stress exponent $n$ and a number of thermally activated terms that define the rate controlling processes for dislocation motion both within subgrains and in sub-boundaries. The model also allows for progressive increase in misorientation of subboundaries during straining, and predicts a small dependence of subgrain size on misorientation. Comparison of the resultant stress-subgrain size equation with published experimental data on a range of materials confirms the likelihood of a dependence on the creep exponent. The influence of the thermally activated terms is likely to be small for metals and alloys.

For minerals, the model predicts that the subgrain size may depend significantly on temperature and chemical environment, and to a lesser extent on sub-boundary misorientation and strain heterogeneity. The quantitative influence of these parameters is not known, preventing use of our theoretical model as an accurate palaeopiezometer at this stage. Preliminary calibration experiments in which the chemical environment is carefully characterized are underway. However, the model does suggest that the existing empirical stress/ subgrain size relations must be used with caution, and that subgrain size palaeopiezometry is unlikely to further improve our knowledge of earth stresses until better experimental data are available.

\section{Acknowledgements}

This work was funded by the U.S. Geological Survey, Earthquake Studies Program, under contract no. 14-08-0001-19797, and we gratefully acknowledge their support.

\section{References}

Amelinckx, S. and Strumane, R. Acta Met. 8, 312 (1960). 
Ashby, M. F. Phil. Mag. 21, 399 (1970).

Barrett, C. R. Trans. Met. Soc. AIME 239, 1726 (1967).

Barrett, C. R., Muehleisen, E. C. and Nix, W. D. Mater. Sci. Eng. 10, 33 (1972).

Barrett, C. R., Nix, W. D. and Sherby, O. D. Trans. ASM 59, 3 (1966).

Bell, T. H. and Etheridge, M. A. Tectonophysics 32, 235 (1976).

Birch, F. (p. 140), In: Clark, Sydney P., Jr. (ed.) Handbook of Physical Constants, The Geological Society of America (1966).

Bird, J. E., Mukherjee, A. K. and Dorn, J. E. In: Brandon, D. G. and Rosen, A. (eds.) Quantitative Relation Between Properties and Microstructure, Israel University Press, Jerusalem. 255-342 (1969).

Blum, W. Phys. Stat. Sol. (b) 45, 561 (1971).

Carter, N. L. and Hansen, F. D. EOS 61, 1131 (1980).

Challenger, K. D. and Moteff, J. Met. Trans. 4, 749 (1973).

Christie, J. M. and Ord, A. J. Geophys. Res. 85, 6253 (1980).

Christie, J. M., Ord, A. and Koch, P. S. EOS Trans. AGU 61, 377 (1980).

Dandekar, D. P. J. Appl. Phys. 39, 3694 (1968).

Etheridge, M. A. and Kirby, S. H. Tectonophysics, in press (1982).

Etheridge, M. A. and Wilkie, J. C., Tectonophysics, 78, 475 (1981).

Evans, H. E. and Knowles, G. Acta Met. 25, 963 (1977).

Exell, S. F. and Warrington, D. H. Phil. Mag. 26, 1121 (1972).

Fields, R. J., Weerasooriya, T. and Ashby, M. F. Cambridge University Engineering Department Report, CUED/C/MATS/TR.55 (1979).

Fine, M. E., Rev. Sci. Instr. 28, 643 (1957).

Friedel, J. Dislocations, Pergamon, London (1964).

Garofalo, F. Trans. Met. Soc. AIME 227, 351 (1963).

Garofalo, F., Richmond, O., Domis, W. F. and von Gemmingen, F. Proc. Inst. Mech. Eng. 178, 1-31 (1963).

Garofalo, F., Whitmore, R. W., Domis, W. F. and von Gemmingen, F. Trans. Met. Soc. AIME 221, 310 (1961).

Gittus, J. H. Phil. Mag. A. 39, 829 (1979).

Gueguen, Y. Geophys. Res. Letters 6, 357 (1979).

Guillope, M. and Poirier, J. P. J. Geophys. Res. 84, 5557 (1979).

Hanks, T. C. Pageoph. 115, 441 (1977).

Harper, J. and Dorn, J. E. Acta Met. 5, 654 (1957).

Hirsch, P. B. Journal de Physique 42, C3, 149 (1981).

Hirth, J. P. and Lothe, J. Theory of Dislocations, McGraw-Hill, New York (1968).

Hobbs, B. E. Tectonophysics 6, 353 (1968).

Hobbs, B. E. Tectonophysics 78, 335 (1981).

Hobbs, B. E. Tectonophysics, in press (1982).

Holt, D. L. J. Appl. Phys. 4, 3197 (1970).

Hornack, P. and Kohlstedt, D. L. EOS Trans. AGU 60, 369 (1979).

Jaoul, O., Froidevaux, C., Durham, W. B. and Michant, M. Earth Plan. Sci. Letters 47, 391 (1980).

Kestenbach, H.-J., Krause, W. and da Silveira, T. L. Acta Met. 26, 661 (1978).

Knipe, R. J. Tectonophysics 64, T11 (1979).

McGarr, A. and Gay, N. C., Ann. Rev. Earth Plan. Sci. 6, 405 (1978).

McQueen, H. J., Wong, W. A. and Jonas, J. J. Canadian J. Phys. 45, 1225 (1967).

Mazza, J. A. and Willoughby, G. J. Iron and Steel Inst. 204, 718 (1966).

Mercier, J. C. C., Anderson, D. A. and Carter, N. L., Pageoph. 115, 199 (1977).

Michel, D. J., Moteff, J. and Lovell, A. J., Acta Met. 21, 1269 (1973).

Nabarro, F. R. N., Theory of Crystal Dislocations, Oxford University Press, Oxford (1967).

Ord, A., Determination of flow stress from microstructures of mylonitic rocks. Unpubl. Ph.D. thesis, U.C.L.A. (1981).

Orlová, A., Tobolová, Z. and Cadek, J. Phil. Mag. 26, 1263 (1972).

Parker, J. D. and Wilshire, B. Phil. Mag. 34, 485 (1976).

Poirier, J. P. Phil. Mag. 26, 713 (1972). 
Poirier, J.-P. and Nicolas, A. J. Geol. 83, 707 (1975).

Raleigh, C. B. and Kirby, S. H. Mineral. Soc. Amer. Spec. Pap. 3, 113 (1970).

Ricoult, D. and Kohlstedt, D. L. EOS Trans. AGU 61, 403 (1980).

Robinson, S. L. and Sherby, O. D. Acta Met. 17, 109 (1969).

Ross, J. V., Ave Lallement, H. G. and Carter, N. L. Tectonophysics 71, 123 (1981).

Schmid, S. M., Paterson, M. S. and Boland, J. N., Tectonophysics 65, 245 (1980).

Sherby, O. D. and Burke, M., Prog. Mat. Sci. 13, 325 (1968).

Sherby, O. D., Klundt, R. H. and Miller, A. K., Met. Trans. A. 8A, 843 (1977).

da Silveira, T. J. and Monteiro, S. N., Proc. Sec. Int. Conf. Mech. Behaviour Mat., Boston. 334 (1976).

Spingarn, J. R. and Nix, W. D. Acta Met. 27, 171 (1979).

Takeuchi, S. and Argon, A. S. J. Mat. Sci. 11, 1542 (1976).

Thompson, A. W. Met. Trans. 8A, 833 (1977).

Twiss, R. J. Pageoph. 115, 227 (1977).

Weathers, M. S., Bird, J. M., Cooper, R. F. and Kohlstedt, D. L. J. Geophys. Res. 84, 7495 (1980).

Weertman, J. J. Appl. Phys. 28, 362 (1957).

Weertman, J. Trans. ASM 61, 680 (1968).

White, S. H. Nature 244, 276 (1973).

White, S. H. Phil. Trans. Royal Soc. Lond. A. 283, 69 (1976).

White, S. H. Tectonophysics 39, 143 (1977).

White, S. H. Contrib. Mineral. Petrol. 70, 193 (1979a).

White, S. H. Bull. Minéral. 102, 75 (1979b).

Young, C. M. and Sherby, O. D. J. Iron and Steel Inst. 211, 640 (1973). 